\title{
Erectile dysfunction - overview from a cardiovascular perspective
}

\author{
Baumann, Frederic ; Hehli, Deborah ; Makaloski, Vladimir ; Schumacher, Martin ; Schönhofen, Heinz ;
} Diehm, Nicolas

\begin{abstract}
Erectile dysfunction (ED) is an evolving health problem with growing incidence in the ageing male population with potentially predictive value for cardiovascular and other chronic diseases. ED shares the common cardiovascular risk factors. The aetiology of ED is numerous including neurogenic, psychogenic, arteriogenic, and venogenic reasons. The origin of arteriogenic ED is frequently atherosclerosis. Patients not adequately responding to conservative measures including oral medication are often referred to further vascular diagnostics and therapy. At present, the refinements in endovascular therapy allow for minimal-invasive revascularization of erection-related arteries. The role of endovascular therapy in the complex framework of the multifactorial causes of ED requires further scientific scrutiny.
\end{abstract}

DOI: https://doi.org/10.1024/0301-1526/a000627

Posted at the Zurich Open Repository and Archive, University of Zurich

ZORA URL: https://doi.org/10.5167/uzh-151440

Journal Article

Accepted Version

Originally published at:

Baumann, Frederic; Hehli, Deborah; Makaloski, Vladimir; Schumacher, Martin; Schönhofen, Heinz; Diehm, Nicolas (2017). Erectile dysfunction - overview from a cardiovascular perspective. Vasa, 46(5):347353.

DOI: https://doi.org/10.1024/0301-1526/a000627 
The Effect of a New Angiographic Imaging Technology on Radiation Dose in Visceral Embolization Procedures

Frederic Baumann, MD ${ }^{1,2}$; Constantino Peña MD ${ }^{1}$, Roman Kloeckner, MD ${ }^{2}$, Barry T Katzen MD ${ }^{1}$; Ripal Gandhi MD ${ }^{1}$; James B Benenati MD ${ }^{1}$

${ }^{1}$ Miami Cardiac \& Vascular Institute, Baptist Hospital, Florida Miami

${ }^{2}$ Clinical and Interventional Angiology, University Hospital of Zurich, Zurich, Switzerland

${ }^{3}$ Interventional Radiology, University Hospital of Mainz, Germany

\section{Corresponding Author}

Frederic Baumann, MD

Miami Cardiac \& Vascular Institute (MCVI)

Baptist Hospital of Miami

8900 N. Kendall Drive

Miami, Florida 33176

Phone: 786-702-9945

FAX: 786-596-2999

E-Mail: fredericbaumann@ hotmail.com 


\title{
The Effect of a New Angiographic Imaging Technology on Radiation Dose in Visceral Embolization Procedures
}

\author{
Abstract \\ Purpose \\ To evaluate the impact of a new angiographic imaging technology on radiation dose \\ during visceral embolization procedures involving both fluoroscopy and digital \\ subtraction angiography.
}

\section{Material and Methods}

A retrospective analysis from a single-center consecutive series of patients was performed comparing two angiographic imaging systems. The AlluraClarity (CIQ [Philips Healthcare, Best, Netherlands]) was used in 100 patients ( $\mathrm{n}=59$ male, mean age: 70.6 years) from July 2013 to April 2014 and compared to the former AlluraXper (AX) technology used in 139 patients ( $n=71$ male, mean age: 70.1 years) from May 2011 to June 2013. Patients were categorized according to body-mass-index (BMI $\left[\mathrm{kg} / \mathrm{m}^{2}\right]$ ) - group 1: BMI $<25$, group 2 : $\mathrm{BMI} \geq 25$ and $<30$, group 3 : $\mathrm{BMI} \geq 30$. Fluoroscopy time, the total dose of iodinated contrast administered and procedural AirKerma (Ka,r: milli Gray [mGy]) were obtained.

\section{Results}

Mean BMI was $26.4 \pm 5.0 \mathrm{~kg} / \mathrm{m}^{2}$ in the CIQ and $26.4 \pm 7.1 \mathrm{~kg} / \mathrm{m}^{2}$ in the AX group $(\mathrm{p}=.93)$. Fluoroscopy time and the amount of contrast media were equally distributed. Ka,r was 1342.9 mGy versus 2214.8 mGy ( $p<.001$, t-test) comparing CIQ to AX. Comparing CIQ to AX, BMI subgroup analysis revealed a mean Ka,r of $970.1 \mathrm{mGy}$ to $1586.1 \mathrm{mGy}$ ( $\mathrm{p}=.003$, t-test), $1484.7 \mathrm{mGy}$ to $2170.1 \mathrm{mGy}(\mathrm{p}=.02$, t-test) and 1848.8 mGy to 3348.9 mGy ( $\mathrm{p}=.001$, t-test $)$ in BMI groups 1, 2 and 3 .

\section{Conclusion}

CIQ technology significantly reduced mean radiation dose by $39.4 \%$ for visceral embolization procedures at comparable fluoroscopy time and contrast media dose. This dose relationship was consistent across all BMI groups.

\section{Key Words}

Radiation reduction, visceral embolization, imaging technology 


\section{Introduction}

A significant number of patients with significant gastrointestinal bleeding can be treated by angiography with selective embolization of the visceral artery branches. However, arterial embolization of visceral arteries requires sophisticated imaging, exposing both operators as well as patients to high radiation dose ${ }^{1}$. Moreover, in obese patients an exponential increase of ionizing radiation is required to assure proper image quality ${ }^{2-4}$. In consequence, concerns have been raised on the radiation exposure of patients and operators, and investigations have been prompted evaluating radiation dose amounts and strategies for possible dose reduction ${ }^{5-8}$.

To address this major concern of radiation exposure, further developments from the imaging technology are required. AlluraClarity ([CIQ]; Philips Healthcare, Best, Netherlands) is intended to acquire equivalent image quality as the former AlluraXper system ([AX]; Philips Healthcare, Best, Netherlands) but for less radiation. CIQ has previously shown to significantly reduce radiation dose for various interventional cardiology-, interventional (neuro-) radiology and endovascular surgery procedures ${ }^{9-16}$. Despite the challenges of comparing different imaging technologies within different settings, a direct comparison of CIQ and AX was performed in several studies, showing no significant loss of image quality ${ }^{9,13,15,16}$. However, the CIQ technology is optimized for each specific acquisition protocol. Therefore, the promising results are not necessarily negotiable for all types of procedures and body locations. Inherently, achievable dose reduction for each type of procedure will be different as each requires a specific acquisition protocol.

The purpose of the present study was to evaluate the potential benefit of CIQ towards dose reduction and its impact on the physician working habits in patients undergoing visceral embolization therapy, since this is a subset of procedures known to necessitate high radiation doses ${ }^{1,2}$. 


\section{Materials and Methods}

\section{Patients and Methods}

This study evaluated a consecutive series of 239 patients (CIQ: $n=100$ [ $n=59$ male, mean age: 70.6 years], $\mathrm{AX}: \mathrm{n}=139$ [ $\mathrm{n}=71$ male, mean age: 70.1 years]) undergoing visceral embolization therapy at a tertiary referral center. The indication for visceral embolization was related to gastrointestinal bleeding in all patients. Information on patients' demographics characteristics including weight and height $(\mathrm{cm})$ and differentiated for CIQ and AX is provided in Table 1. Since physical conditions were shown to affect image acquisition and quality ${ }^{2-4}$, body-mass-index (BMI $\left[\mathrm{kg} / \mathrm{m}^{2}\right]$ ) was evaluated. According to the BMI values, patients were categorized into three groups: group 1: BMI $<25 \mathrm{~kg} / \mathrm{m}^{2}$, group 2: BMI $\geq 25$ to $<30 \mathrm{~kg} / \mathrm{m}^{2}$ and group $3: \mathrm{BMI} \geq 30 \mathrm{~kg} / \mathrm{m}^{2}$. As outlined in Table 1, there was a homogenous BMI distribution comparing the CIQ and AX groups.

To estimate the benefit of the novel CIQ technology, radiation dose recordings using CIQ were compared to radiation dose recordings using the former AX technology (control group). AX measurements were obtained from May 2011 to June 2013 and CIQ measurements after installation in July 2013 from July 2013 until April 2014, respectively. All procedures were performed following the radiation safety principle of As Low As Reasonably Achievable for both imaging technologies. Data collection and analysis was performed retrospectively. In line, the interventionists were not aware that this data is going to be evaluated at the time of procedure. Approval from our institutional IRB was obtained including a waiver of informed consent due to the retrospective nature of the evaluation. All data were collected in a Health Insurance Portability and Accountability Act compliant manner.

\section{CIQ Technology and Measurements Obtained}

CIQ is intended to acquire the equivalent image quality as $\mathrm{AX}$, but for less radiation. This is achieved by an improved real-time image noise reduction algorithm as well as hardware optimization. The algorithm utilizes real-time automatic pixel shift, motion compensation, temporal- and spatial noise reduction to preserve image quality, while associated hardware reconfiguration is intended to reduce the entrance dose by adjusting and optimizing the full acquisition chain for different anatomic regions ${ }^{9}$.

For the purpose of comparing the performance of the former AX with the novel CIQ technology, information on fluoroscopy time (minutes), total dose of iodinated contrast and AirKerma (Ka,r [milli Gray: mGy]) were evaluated. The Ka,r was calculated for the patient entrance reference point, which was considered the approximation of the patient's skin, located in the central X-ray beam and $15 \mathrm{~cm}$ from the isocenter towards the focal spot ${ }^{17}$. Fluoroscopy time and contrast usage served as indirect parameters for image quality. Similar amounts of contrast and fluoroscopy time were considered to indicate equivalent image quality comparing AX and CIQ. However, no direct comparison of image quality was performed.

\section{Statistical Analysis}

Categorical variables are presented as number and percentage and are compared using the Fisher's exact test. Continuous variables are presented as means \pm standard 
deviation $( \pm \mathrm{SD})$ and $95 \%$ confidence interval $(\mathrm{CI})$ where appropriate, and are compared using an independent t-test after checking for homogeneous distribution. This was done for the comparison of the total study and control groups, as well as for BMI subgroup analysis. A p-value $<0.05$ was considered statistically significant. Statistical calculations were performed using STATA software (STATA Statistics version 14.0, StataCorp, College Station, Texas). 


\section{Results}

A total of 239 patients were undergoing visceral embolization procedures. As outlined in Table 1, patients did not differ by age, gender distribution and BMI when comparing the CIQ and AX cohorts. Procedural details are listed in Table 2 showing longer procedure times in CIQ patients $(108.3 \pm 45.3 \mathrm{~min}$ versus $91.4 \pm 44.0 \mathrm{~min}, \mathrm{p}=0.004, \mathrm{t}-$ test). Figure 1 illustrates BMI subgroup analysis showing a difference in the BMI group 2 (117.0 \pm 7.1 min versus $86.8 \pm 5.0$ min, $\mathrm{p}<0.001$, t-test).

Fluoroscopy time $(24.7 \pm 15.9$ min versus $24.8 \pm 27.3 \mathrm{~min}, \mathrm{p}=.96$, t-test) and the use of contrast media volume $(137.9 \pm 60.2$ versus $141.4 \pm 62.1, \mathrm{p}=.67$, t-test $)$ were similar when comparing the CIQ and AX groups and showing no significant differences in BMI subgroup analysis.

The overall Ka,r was lower in the CIQ group compared to the AX group: $1342.9 \pm 1080.1 \mathrm{mGy}(95 \%$-CI: 1128.6 to 1557.2$)$ versus $2214.8 \pm 1826.8$ (95\%-CI: 1908.4 to $2421.2, \mathrm{p}<.001$, t-test).

Accordingly, mean Ka,r was $970.1 \pm 847.2 \mathrm{mGy}(95 \%$-CI: 702.7 to 1237.5$)$ versus $1586.1 \pm 1335.0 \mathrm{mGy}(95 \%-\mathrm{CI}$ : 1241.2 to 1930.9$)$ comparing CIQ versus AX (p=.003, ttest) in BMI group 1, $1484.7 \pm 1160.6 \mathrm{mGy}(95 \%$-CI: 1113.5 to 1855.9$)$ versus 2170.1 $\pm 1458.2 \mathrm{mGy}(95 \%$-CI: 1726.7 to 2613.4$)$ in BMI group $2(\mathrm{p}=.02$, t-test) and $1848.8 \pm 1128.0 \mathrm{mGy}(95 \%$-CI: 1305.1 to 2392.5$)$ versus $3348.9 \pm 2395.7 \mathrm{mGy}(95 \%$-CI: 2526.0 to 4171.9 ) in BMI group 3 ( $\mathrm{p}=.001$, t-test), respectively (Figure 2). 


\section{Discussion}

There has been a rapid growth of endovascular therapies necessitating radiation within the last decades ${ }^{18,19}$. In line, operators have been faced with continued exposure and increasing cumulative radiation dose exposure ${ }^{18,19}$. This development and its consequences are a major concern not only for patients but also for the operators involved in angiographic interventional settings ${ }^{5,7,8,20,21}$. As a result, efforts to effectively reduce radiation dose while maintaining the necessary level of image quality have become paramount ${ }^{22}$. However, specific procedures such as visceral interventions as well as patients with higher body weight and BMI require more radiation to maintain image quality ${ }^{2}$. Therefore, technical advancements are necessitated to reduce radiation for these patients and procedures. The present study showed an average dose reduction of $39.4 \%$ in patients treated with CIQ when compared to AX imaging technology and this finding held true for all BMI groups.

Despite the challenges of comparing different imaging technologies within different settings, CIQ was previously shown to reduce radiation dose for no significant loss of image quality when compared to $\mathrm{AX}^{9,10,15,16}$. However, Soderman et al. were the only one performing a 1:1 direct comparison of the image quality in an interventional neuroradiology setting ${ }^{9}$. In each patient imaging was obtained using the CIQ and AX technologies. The images were reviewed in a randomized and blinded manner by three neuroradiologists and thereby, no loss of image quality was observed but the CIQ images were acquired using $25 \%$ of the $\mathrm{AX}$ radiation dose, only.

Thereafter, multiple single center series evaluated the potential of CIQ in reducing radiation dose for various types of procedures. Overall, a radiation dose reduction of $>40 \%$ was achieved in all ${ }^{10-13,15,16}$. However, there was a wide range of radiation dose reduction indicating that the benefit of CIQ varies according to the procedure type and patients' habitus.

In order to properly generalize and to compare both imaging technologies in this study, the assessment of fluoroscopy time and the amount of contrast media utilized served as indirect parameters to assess procedural complexity between the two groups. The relatively large number of patients in both groups is also important to help compare the groups. Fluoroscopy time and the amount of contrast media used were similar in both groups (Table 2). Therefore, we suggest that the procedural complexity was comparable as indicated by the fluoroscopy time and the amount of contrast used, which we account as objective parameters.

The present study has several limitations. First, the current analysis is based on a retrospective evaluation of a de-identified dataset. Therefore, it was not possible to review specific procedural protocols and angiograms. Nevertheless, the heterogeneous patient population and procedural complexity in this large study population allows for indirect comparison of the two imaging modalities. In addition, it was not possible to systematically compare the image quality of CIQ and AX technology.

Second, there were a variety of different visceral embolization procedures included within the present study limiting the interpretation of procedure time and radiation dose. 


\section{Conclusion}

The present study demonstrated that radiation dose can be significantly reduced in patients undergoing visceral embolization procedures by the use of CIQ. As expected, the results show an increase in radiation dose correlating with patient size. Despite the variability of procedure complexity, a consistent dose reduction was identified across the range of patient BMI. The need to understand the amount of radiation dose reduction possible with new technological advances is important as we attempt to determine which changes are truly significant while maintaining image and procedural quality. Further study of these technological advances is warranted especially for different acquisition protocols as the number and complexity of angiographic interventional procedures continue to increase.

\section{Acknowledgments}

None

\section{Conflict of interest}

Author \#4 is a Member of the Advisory Board of Philips but having no direct conflict of interest to report with this study.

All other authors report neither financial nor industrials relationships nor a conflict of interest. 


\section{References}

1. Miller DL, Balter S, Cole PE, Lu HT, Schueler BA, Geisinger M, Berenstein A, Albert R, Georgia JD, Noonan PT, Cardella JF, St George J, Russell EJ, Malisch TW, Vogelzang RL, Miller GL, 3rd, Anderson J, study R-I. Radiation doses in interventional radiology procedures: the RAD-IR study: part I: overall measures of dose. Journal of vascular and interventional radiology: JVIR 2003;14:711-727.

2. Ketteler ER, Brown KR. Radiation exposure in endovascular procedures. Journal of vascular surgery 2011;53:35S-38S.

3. Shah A, Das P, Subkovas E, Buch AN, Rees M, Bellamy C. Radiation Dose During Coronary Angiogram: Relation to Body Mass Index. Heart, lung \& circulation 2014.

4. Bryk SG, Censullo ML, Wagner LK, Rossman LL, Cohen AM. Endovascular and interventional procedures in obese patients: a review of procedural technique modifications and radiation management. Journal of vascular and interventional radiology : JVIR 2006; 17:27-33.

5. Vano E, Gonzalez L, Guibelalde E, Fernandez JM, Ten JI. Radiation exposure to medical staff in interventional and cardiac radiology. The British journal of radiology 1998;71:954-960.

6. Pitton MB, Kloeckner R, Schneider J, Ruckes C, Bersch A, Duber C. Radiation exposure in vascular angiographic procedures. Journal of vascular and interventional radiology : JVIR 2012;23:1487-1495.

7. Miller DL, Balter S, Dixon RG, Nikolic B, Bartal G, Cardella JF, Dauer LT, Stecker MS, Society of Interventional Radiology Standards of Practice C. Quality improvement guidelines for recording patient radiation dose in the medical record for fluoroscopically guided procedures. Journal of vascular and interventional radiology : JVIR 2012;23:11-18.

8. Stecker MS, Balter S, Towbin RB, Miller DL, Vano E, Bartal G, Angle JF, Chao CP, Cohen AM, Dixon RG, Gross K, Hartnell GG, Schueler B, Statler JD, de Baere T, Cardella JF, Safety SIR, Health C, Committee CSoP. Guidelines for patient radiation dose management. Journal of vascular and interventional radiology: JVIR 2009;20:S263-273.

9. Soderman M, Holmin S, Andersson T, Palmgren C, Babic D, Hoornaert B. Image noise reduction algorithm for digital subtraction angiography: clinical results. Radiology 2013;269:553-560.

10. Racadio J, Strauss K, Abruzzo T, Patel M, Kukreja K, Johnson N, den Hartog M, Hoornaert B, Nachabe R. Significant dose reduction for pediatric digital subtraction angiography without impairing image quality: preclinical study in a piglet model. AJR American journal of roentgenology 2014;203:904-908.

11. Dekker LR, van der Voort PH, Simmers TA, Verbeek XA, Bullens RW, Veer MV, Brands PJ, Meijer A. New image processing and noise reduction technology allows reduction of radiation exposure in complex electrophysiologic interventions while maintaining optimal image quality: a randomized clinical trial. Heart rhythm : the official journal of the Heart Rhythm Society 2013;10:1678-1682.

12. Soderman M, Mauti M, Boon S, Omar A, Marteinsdottir M, Andersson T, Holmin $\mathrm{S}$, Hoornaert B. Radiation dose in neuroangiography using image noise reduction technology: a population study based on 614 patients. Neuroradiology 2013;55:1365-1372. 
13. Schernthaner RE, Duran R, Chapiro J, Wang Z, Geschwind JF, Lin M. A new angiographic imaging platform reduces radiation exposure for patients with liver cancer treated with transarterial chemoembolization. European radiology 2015.

14. Sailer AM, Schurink GW, Bol ME, de Haan MW, van Zwam WH, Wildberger JE, Jeukens CR. Occupational Radiation Exposure During Endovascular Aortic Repair. Cardiovascular and interventional radiology 2015;38:827-832.

15. Eloot L, Thierens H, Taeymans Y, Drieghe B, De Pooter J, Van Peteghem S, Buytaert D, Gijs T, Lapere R, Bacher K. Novel X-ray imaging technology enables significant patient dose reduction in interventional cardiology while maintaining diagnostic image quality. Catheterization and cardiovascular interventions : official journal of the Society for Cardiac Angiography \& Interventions 2015.

16. van Strijen MJ, Grunhagen T, Mauti M, Zahringer M, Gaines PA, Robinson GJ, Railton NJ, van Overhagen H, Habraken J, van Leersum M. Evaluation of a noise reduction imaging technology in iliac digital subtraction angiography: noninferior clinical image quality with lower patient and scatter dose. Journal of vascular and interventional radiology: JVIR 2015;26:642-650 e641.

17. Commission. IE. Medical electrical equipment: part 2-43-particular requirements for the safety of x-ray equipment for interventional procedures. Geneva, Switzerland: International Electrotechnical Commission, 2010. 2010;2nd edition:243.

18. Duncan JR, Balter S, Becker GJ, Brady J, Brink JA, Bulas D, Chatfield MB, Choi S, Connolly BL, Dixon RG, Gray JE, Kee ST, Miller DL, Robinson DW, Sands MJ, Schauer DA, Steele JR, Street M, Thornton RH, Wise RA. Optimizing radiation use during fluoroscopic procedures: proceedings from a multidisciplinary consensus panel. Journal of vascular and interventional radiology : JVIR 2011;22:425-429.

19. Mettler FA, Jr., Bhargavan M, Faulkner K, Gilley DB, Gray JE, Ibbott GS, Lipoti JA, Mahesh M, McCrohan JL, Stabin MG, Thomadsen BR, Yoshizumi TT. Radiologic and nuclear medicine studies in the United States and worldwide: frequency, radiation dose, and comparison with other radiation sources--1950-2007. Radiology 2009;253:520-531.

20. Miller DL, Vano E, Bartal G, Balter S, Dixon R, Padovani R, Schueler B, Cardella JF, de Baere T, Cardiovascular, Interventional Radiology Society of E, Society of Interventional $\mathrm{R}$. Occupational radiation protection in interventional radiology: a joint guideline of the Cardiovascular and Interventional Radiology Society of Europe and the Society of Interventional Radiology. Journal of vascular and interventional radiology : JVIR 2010;21:607-615.

21. Mettler FA, Jr., Thomadsen BR, Bhargavan M, Gilley DB, Gray JE, Lipoti JA, McCrohan J, Yoshizumi TT, Mahesh M. Medical radiation exposure in the U.S. in 2006: preliminary results. Health physics 2008;95:502-507.

22. Le Heron J, Padovani R, Smith I, Czarwinski R. Radiation protection of medical staff. European journal of radiology 2010;76:20-23. 


\section{Legends of Figures}

\section{Figure 1}

Depiction of procedure and fluoroscopy times according to BMI categories.

Figure 2

Ka,r values for the three different BMI groups. 


\section{Table 1}

Patients' characteristics and demographics of 239 patients undergoing visceral embolization

\begin{tabular}{|l|c|c|c|}
\hline & $\begin{array}{c}\text { AX } \\
\mathbf{n = 1 3 9}\end{array}$ & $\begin{array}{c}\text { CIQ } \\
\mathbf{n = 1 0 0}\end{array}$ & p-Value \\
\hline Male, $\mathrm{n}(\%)$ & $71(51.1)$ & $59(59.0)$ & .24 \\
\hline Age $[$ years], mean $( \pm$ SD) & $70.1( \pm 16.3)$ & $70.6( \pm 13.8)$ & .80 \\
\hline BMI [kg/m $\left.{ }^{2}\right]$, mean $( \pm$ SD) & $26.4( \pm 7.1)$ & $26.4( \pm 5.0)$ & .93 \\
\hline BMI group 1, n $(\%)$ & $60(43.2)$ & $41(41.0)$ & .79 \\
\hline BMI group 2, n (\%) & $44(31.6)$ & $40(40.0)$ & .22 \\
\hline BMI group 3, n $(\%)$ & $35(25.2)$ & $19(19.0)$ & .28 \\
\hline
\end{tabular}

AX: AlluraXper, CIQ: AlluraClarity, n: number, SD: standard deviation, kg: kilogramm, m: meter

BMI group 1: BMI $<25 \mathrm{~kg} / \mathrm{m}^{2}$, BMI group 2: $\mathrm{BMI} \geq 25 \mathrm{~kg} / \mathrm{m}^{2}$ and $<30 \mathrm{~kg} / \mathrm{m}^{2}$, BMI group 3: $\mathrm{BMI} \geq 30 \mathrm{~kg} / \mathrm{m}^{2}$ 
Table 2

Procedural details of 239 patients undergoing visceral embolization.

\begin{tabular}{|l|c|c|c|}
\hline & $\begin{array}{c}\text { AX } \\
\mathbf{n = 1 3 9}\end{array}$ & $\begin{array}{c}\text { CIQ } \\
\mathbf{n = 1 0 0}\end{array}$ & p-Value \\
\hline Procedure time [min], mean $( \pm \mathrm{SD})$ & $91.4( \pm 44.0)$ & $108.3( \pm 45.3)$ & .004 \\
\hline Flouro time [min], mean $( \pm \mathrm{SD})$ & $24.8( \pm 27.3)$ & $24.7( \pm 15.9)$ & .96 \\
\hline Contrast [cc], mean $( \pm \mathrm{SD})$ & $141.4( \pm 62.1)$ & $137.9( \pm 60.2)$ & .67 \\
\hline Air Kerma [mGy], mean $( \pm \mathrm{SD})$ & $2214.8( \pm 1826.8)$ & $1342.9( \pm 1080.2)$ & $<.001$ \\
\hline Air Kerma [mGy] PFM [min], mean $( \pm \mathrm{SD})$ & $103.2( \pm 65.6)$ & $61.8( \pm 50.0)$ & $<.001$ \\
\hline
\end{tabular}

AX: AlluraXper, CIQ: AlluraClarity, n: number, min: minutes, SD: standard deviation, cc: centiliter, mGy: mili Gray, PFM: per flouroscopic minute 